\title{
Conexões
}

Educação Física, Esporte e Saúde

\section{0 ciclo menstrual tem influência sob a flexibilidade de mulheres treinadas e sedentárias? um estudo piloto}

\author{
Isadora Cristina Ribeiro ${ }^{1}$ \\ Carlos Roberto Padovani ${ }^{2}$ \\ João Paulo Borin ${ }^{1}$
}

\section{RESUMO}

Introdução: A literatura acerca do desempenho da flexibilidade e sua relação com o ciclo menstrual é controversa, uma vez que as pesquisas avaliam diferentes populações. Objetivo: comparar o desempenho físico entre mulheres sedentárias e treinadas para a capacidade de flexibilidade durante as fases de dois ciclos menstruais e verificar se o desempenho de fases iguais sofre alterações de um ciclo para o outro. Metodologia: durante dois ciclos menstruais 12 voluntárias divididas nos grupos treinadas: $G 1(n=6)$ e sedentárias: $G 2(n=6)$ participaram de um protocolo de treinamento específico para flexibilidade e foram avaliadas quanto ao desempenho em flexibilidade pelo teste de sentar e alcançar no banco de Wells a cada fase dos ciclos menstruais. Resultados e discussão: $O \mathrm{G} 1$ obteve maior desempenho do que o $\mathrm{G} 2 \mathrm{em}$ todas as fases analisadas. Não houve diferença no desempenho entre as fases dos ciclos menstruais avaliados para nenhum dos grupos. Além disto, o desempenho entre fases iguais nos diferentes ciclos manteve-se estável. Conclusão: mulheres treinadas em flexibilidade tem melhor desempenho nessa capacidade motora do que mulheres sedentárias e que o desempenho em flexibilidade não é afetado pelo ciclo menstrual em mulheres treinadas em flexibilidade e sedentárias.

Palavras-chave: Aptidão física. Ciclo ovariano. Exercício de alongamento muscular.

\footnotetext{
1 Universidade Estadual de Campinas.

2 Universidade Estadual Paulista.
}

\section{Correspondência:}

Isadora Cristina Ribeiro. Faculdade de Ciências Médicas, Universidade Estadual de Campinas, Rua Tessália Vieira de Camargo, 126. Cidade Universitária Zeferino Vaz. CEP 13083-887. Campinas, $\mathrm{SP}$, Brasil. 


\section{Does the menstrual cycle influence the flexibility of trained and sedentary women? a pilot study}

\section{ABSTRACT}

Introduction: The literature on the performance of flexibility and its relationship to the menstrual cycle is controversial, as research evaluates different populations. Objective: this study aimed to compare physical performance among sedentary women trained for flexibility during the phases of two menstrual cycles and to verify if the performance of equal phases changes from one cycle to another. Methodology: during two menstrual cycles 12 volunteers divided into trained groups: $G 1(n=6)$ and sedentary: $G 2(n=6)$ participated in a flexibility specific training protocol and were evaluated for flexibility performance by the test of sit and reach in the Wells bank at each phase of the menstrual cycles. Results and discussion: G1 had better performance than $\mathrm{G} 2$ in all phases analyzed. There was no difference in performance between the phases of the menstrual cycles evaluated for either group, and the performance between equal phases in the different cycles remained stable. Conclusion: women trained in flexibility perform better in this motor capacity than sedentary women and that the performance in flexibility is not affected by the menstrual cycle in women trained in flexibility and sedentary.

Keywords: Physical Fitness. Ovarian Cycle. Muscle Stretching Exercise.

\section{¿El ciclo menstrual influye en la flexibilidad de las mujeres entrenadas y sedentarias? un estudio piloto}

\section{RESUMEN}

Introducción: La literatura sobre el desempeño de la flexibilidad y su relación con el ciclo menstrual es controvertida, ya que la investigación evalúa diferentes poblaciones. Objetivo: este estudio tuvo como objetivo comparar el rendimiento físico entre mujeres sedentarias entrenadas para la flexibilidad durante las fases de dos ciclos menstruales y para verificar si el rendimiento de fases iguales cambia de un ciclo a otro. Metodología: durante dos ciclos menstruales, 12 voluntarios se dividieron en grupos entrenados: G1 ( $\mathrm{n}$ $=6)$ y sedentarios: $G 2(n=6)$ participaron en un protocolo de entrenamiento específico de flexibilidad y se evaluó su rendimiento de flexibilidad mediante una prueba sentada. y llegar al banco de Wells en cada fase de los ciclos menstruales. Resultado y discusión: G1 tuvo un mejor rendimiento que G2 en todas las fases analizadas. No hubo diferencias en el rendimiento entre las fases de los ciclos menstruales evaluados para ninguno de los grupos, y el rendimiento entre las fases iguales en los diferentes ciclos se mantuvo estable. Conclusión: las mujeres capacitadas en flexibilidad se desempeñan mejor en esta capacidad motora que las mujeres sedentarias y que el rendimiento en flexibilidad no se ve afectado por el ciclo menstrual en las mujeres capacitadas en flexibilidad y sedentarias.

Palabras clave: Aptitud Física. Ciclo ovárico. Ejercicio de estiramiento muscular. 


\section{INTRODUÇÃO}

Atualmente com o aumento dos estudos relacionados às mulheres e ao funcionamento do corpo humano em relação à atividade física muito tem se pesquisado sobre os diferentes tipos de exercícios e a ação dos hormônios femininos sobre seu desempenho físico. Nesse sentido, estudos relacionados ao ciclo menstrual têm se mostrado interessantes para a compreensão do desempenho feminino em diferentes capacidades motoras, uma vez que este processo promove alterações hormonais que podem influenciar nas respostas corporais frente ao exercício físico (LEBRUN; RUMBALL, 2001).

O comportamento da capacidade de flexibilidade ao longo das fases do ciclo menstrual é ainda controverso na literatura. Alguns autores apontam para a não influência das alterações hormonais das fases do ciclo menstrual sobre o desempenho em flexibilidade (CHAVES; SIMÃO; ARAÚJO, 2002, MELEGARIO et al., 2006; TEIXEIRA et al., 2012). Enquanto Bisi et al. (2009) sugerem um menor desempenho em flexibilidade na fase ovulatória do ciclo menstrual.

As divergências metodológicas dos achados literários podem ter gerado resultados distintos. Além do método de avaliação da flexibilidade distinguir-se entre estudos, as populações avaliadas também variam podendo interferir nos resultados encontrados. Sendo assim, a comparação entre diferentes grupos, selecionados por nível de treinamento, e a avaliação de mais de um ciclo menstrual, compreendendo o processo de forma periódica, podem contribuir com o entendimento do desempenho em flexibilidade nas diferentes fases do ciclo menstrual.

A partir de tais informações o objetivo deste estudo foi comparar o desempenho físico de mulheres com diferentes níveis de treinamento para a capacidade de flexibiidade durante as fases de dois ciclos menstruais. Além disso, verificar se o desempenho tem o mesmo comportamento em fases iguais de ciclos diferentes.

\section{MÉTODO}

Foram avaliadas 12 mulheres com idade média de 20,6 $\pm 3,7$ anos, que faziam uso de anticoncepcional oral há no mínimo seis meses e foram divididas em dois grupos: grupo treinado ( $\mathrm{G} 1$ : treinadas em flexibilidade há no mínimo seis meses) e grupo iniciante (G2: sedentárias há no mínimo seis meses). A coleta de dados só teve início após a aprovação do comitê de ética e pesquisa local e após o esclarecimento acerca da pesquisa, leitura e assinatura do termo de consentimento livre e esclarecido por todas as participantes. 
Para os critérios de inclusão utilizou-se: ser do sexo feminino, fazer o uso de anticoncepcional oral há no mínimo seis meses, já ser treinada em flexibilidade há no mínimo seis meses ou não ser praticante de atividades físicas e ter disponibilidade de iniciar. E para os critérios de exclusão: possuir doenças cardiovasculares, neuromusculares, patologias em tratamento ou qualquer problema de saúde que afete no treinamento, no ciclo menstrual ou que coloque a voluntária em risco.

Em um período de dois meses (oito semanas consecutivas) as voluntárias dos dois grupos fizeram um protocolo de treinamento para flexibilidade, o qual consistia em duas sessões semanais de treinamento de alongamento estático para os músculos isquiotibiais. Para a realização deste protocolo manteve-se um volume equalizado ao longo dos dois ciclos menstruais: três séries de 30 segundos para o exercício de sentar e alcançar os pés com pausa fixa entre as séries de um minuto, como proposto por Gama et al. (2007). O treinamento teve como objetivo a melhora do alcance à frente na maior distância possível.

Além disso, para monitorar o desempenho em flexibilidade fizeram um teste funcional a cada fase de seu ciclo menstrual (no meio da fase), totalizando três vezes ao mês e seis dias de teste de desempenho, completando dois ciclos menstruais (oito semanas). O primeiro teste foi feito na fase folicular (entre 0 terceiro e o quinto dia do fluxo menstrual), o segundo na fase ovulatória (entre o nono e o $10^{\circ}$ dia do ciclo menstrual) e o terceiro na fase lútea (entre 170 e o $21^{\circ}$ dia do fluxo menstrual), como sugerido por Loureiro et al. (2011). Durante o período de análise as voluntárias fizeram somente os exercícios propostos pelo projeto.

O teste funcional aplicado foi o teste do banco de Wells, também conhecido como teste de sentar e alcançar, no qual é medida a amplitude do movimento dos músculos posteriores do tronco e da coxa (WELLS; DILLON, 1952). A voluntária sentou-se em um colchonete com a articulação do joelho totalmente estendida e os pés encostados no equipamento. Para realização do teste projetava-se para frente até onde fosse possível e deslizava os dedos ao longo da régua sem flexionar os joelhos sustentando a posição por pelo menos dois segundos. A média da distância alcançada em três tentativas representou o escore final.

Os dias de aplicação de testes foram sempre seguindo o ciclo menstrual de cada voluntária, de forma individualizada e separadamente dos dias de treinamento. O ciclo menstrual foi determinado e controlado pelo uso da pílula anticoncepcional, uma vez que não há diferenças entre o desempenho em flexibilidade de usuárias ou não de anticoncepcionais orais (TEIXEIRA et al., 2012). 


\section{ANÁLISE Estatística}

Os dados referentes ao desempenho em flexibilidade obtidos pelo teste do banco de Wells foram comparados entre os diferentes grupos, entre as seis fases do período avaliado e entre as mesmas fases dos dois diferentes ciclos menstruais. Foi utilizada a técnica de análise de variância para o modelo de medidas repetidas em grupos independentes complementada com o teste de comparações múltiplas de Bonferroni (JOHNSON; WICHERN, 2007) e considerado significativo o valor de $p<0,05$.

\section{Resultados E Discussão}

Os resultados são apresentados quanto a comparação de desempenho entre os grupos ao longo do período avaliado, quanto ao desempenho nas fases dos dois ciclos menstruais para diferentes grupos e quanto a comparação entre fases iguais ao longo dos dois ciclos menstruais avaliados.

Em relação ao desempenho físico entre os grupos avaliados observou-se que em todas as fases o desempenho do $\mathrm{G} 1$ foi significativamente maior do que do G2 como mostra o gráfico 1 .

\section{DESEMPENHO DOS GRUPOS G1 E G2}

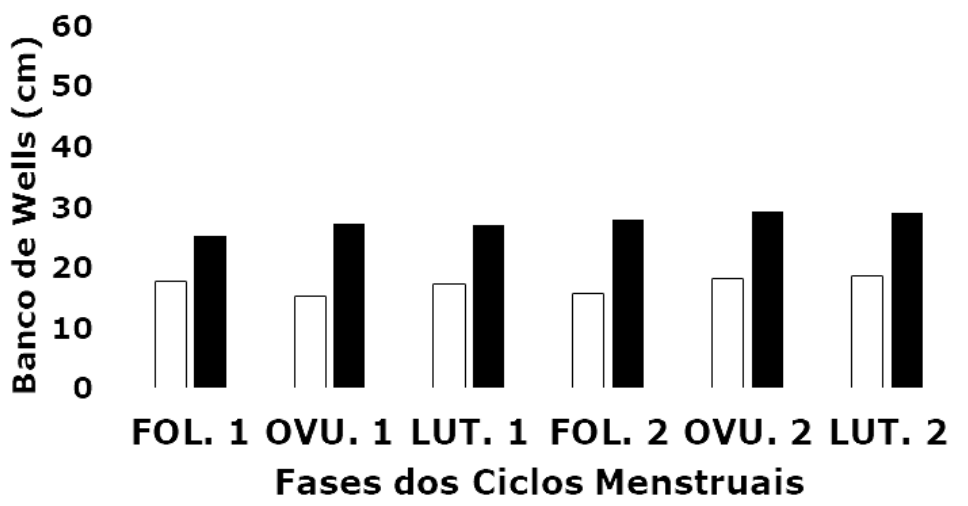

Gráfico 1. Comparação da média de desempenho em flexibilidade entre os grupos treinado (G1) e iniciante (G2). Em todas as fases o grupo G1 apresentou valores significativamente mais altos do que $G 2$, considerando $p<0,05$.

Ao comparar o desempenho entre as fases dos dois ciclos menstruais para os grupos treinado em flexibilidade (G1, gráfico 2A) e grupo sedentário (G2, gráfico 2B) não foram encontradas diferenças significativas para nenhum dos grupos. 


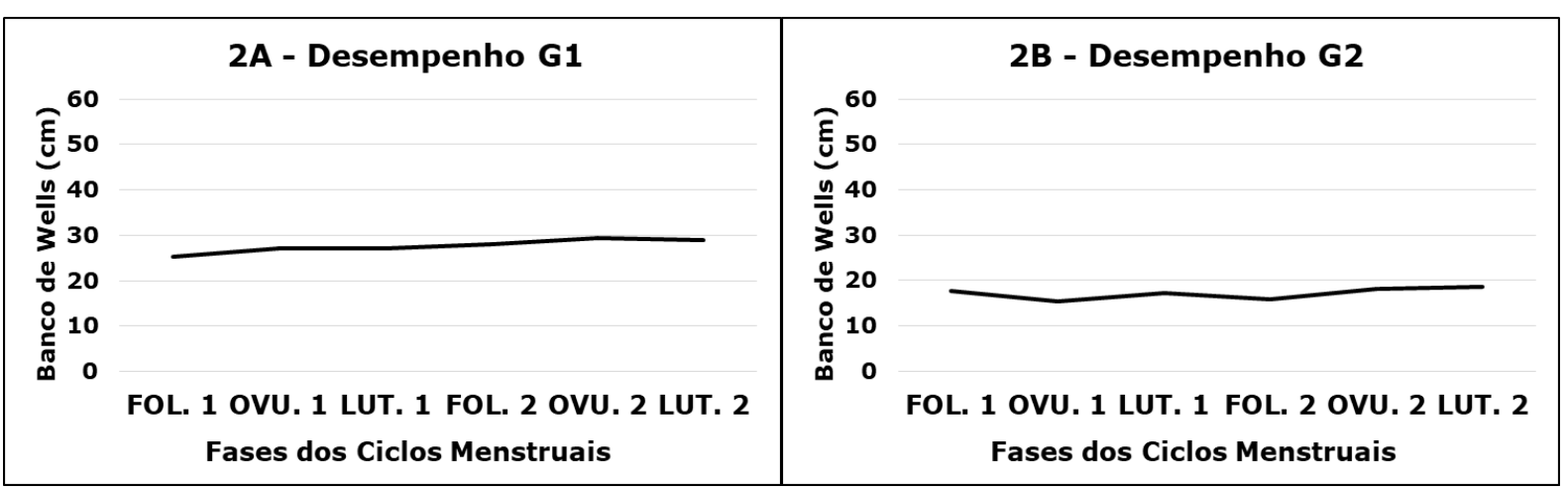

Gráficos 2A e 2B - Média do desempenho em flexibilidade em $\mathrm{cm}$ ao longo das fases de dois ciclos menstruais. A - Desempenho do grupo treinado em flexibilidade (G1) e B Desempenho do grupo sedentário (G2).

Além disso, na comparação entre fases iguais nos dois ciclos analisados (fase folicular 1 comparada com a folicular 2, ovulatória 1 com ovulatória 2 e lútea 1 com lútea 2) nossos resultados apontam para uma melhora no desempenho da flexibilidade $(p>0,05)$ não significativa entre fases iguais dos dois diferentes ciclos para ambos os grupos.

Este estudo destaca-se na comparação do desempenho físico em flexibilidade entre mulheres treinadas e sedentárias, na avaliação de mais de um ciclo menstrual, na comparação de fases iguais entre dois ciclos menstruais consecutivos e na aplicação de um protocolo de treinamento controlado.

O desempenho do grupo treinado (G1) foi maior do que o desempenho do grupo sedentário (G2) em todas as fases avaliadas devido ao nível de treinamento em relação a essa capacidade motora, uma vez que as voluntárias treinadas já tinham a flexibilidade desenvolvida pela prática semanal de aulas de dança (duas vezes na semana) e as voluntárias sedentárias iniciaram a prática desta atividade física no protocolo de treinamento proposto por este projeto. Dessa forma, o nível de aptidão física justifica a diferença no desempenho entre os grupos.

Os resultados aqui apresentados se aproximam dos resultados encontrados por Chaves, Simão e Araújo (2002), Melegario et al. (2006) e Teixeira et al. (2012). Estes estudos além de não encontrarem diferenças significativas no desempenho de flexibilidade entre as fases do ciclo menstrual como observado em nosso estudo, se assemelham à nossa investigação na avaliação do ciclo menstrual em três fases.

Teixeira et al. (2012) investigaram 44 voluntárias fisicamente ativas em flexibilidade através do teste de sentar e alcançar no banco de Wells, assim como em nosso estudo. Entretanto, o objetivo principal do estudo era avaliar se o uso ou não de anticoncepcionais afetava o desempenho de flexibilidade ao longo do ciclo menstrual. Como resultado, encontraram que as diferentes fases 
do ciclo menstrual não interferem na flexibilidade, independente do uso ou não de anticoncepcionais.

Com o mesmo objetivo proposto pelo presente estudo, porém utilizando diferentes métodos de investigação, Melegario et al. (2006) avaliaram 20 mulheres treinadas em ginástica de academia através de um goniômetro em oito movimentos (flexão e abdução da articulação do ombro, flexão da articulação de cotovelo, flexão, extensão e abdução da articulação do quadril, flexão da articulação do joelho e flexão da coluna lombar). E Chaves, Simão e Araújo (2002) avaliaram a flexibilidade de 15 mulheres fisicamente ativas pelo Flexiteste, que avalia a flexibilidade global através de 20 movimentos que envolvem as articulações do tornozelo, joelho, quadril, tronco, punho, cotovelo e ombro.

Cabe destacar que apesar de apresentarem resultados semelhantes, as investigações de Chaves, Simão e Araújo (2002), Melegario et al. (2006) e Teixeira et al. (2012) diferem-se de nosso estudo por não avaliarem mais de um ciclo menstrual. Além disso, não possuíam um protocolo de treinamento controlado para essa capacidade motora e não tinham como objetivo comparar o desempenho entre grupos de diferentes níveis de treinamento.

Em contrapartida a estes achados, Bisi et al. (2009) investigaram o desempenho de flexibilidade através do flexímetro e também do teste de sentar e alcançar no banco de Wells. Entretanto, avaliou o ciclo menstrual em apenas duas fases, descritas como ovulatória e anovulatória e também diferiu-se quanto aos participantes avaliados, composta por 10 mulheres atletas de handebol. Não houve diferenças significativas entre as fases pelo teste banco de Wells, corroborando com nossos resultados e com os resultados apresentados por Teixeira et al. (2012). Apesar disso, no desempenho do teste flexímetro percebeu-se uma redução significativa de desempenho na fase ovulatória.

Nossos resultados juntamente com os estudos de Chaves, Simão e Araújo (2002), Melegario et al. (2006) e Teixeira et al. (2012) sugerem que independentemente do nível de treinamento da população avaliada (fisicamente ativa, treinada ou sedentária), do método de avaliação utilizado para medir a flexibilidade (flexiteste), goniômetro ou banco de Wells) e da presença ou não de um período de treinamento controlado, o ciclo menstrual não tem influência sobre o desempenho dessa capacidade motora.

Já Bisi et al. (2009), por investigar uma população não avaliada anteriormente nesse sentido, sugerem que sujeitos em nível atlético sofrem influência do ciclo menstrual sobre a capacidade de flexibilidade quando medida por flexímetro. Porém, como sugere seu resultado do teste do banco de Wells, essa população também pode não ser afetada pelas alterações hormonais do ciclo menstrual. Nesse sentido novos estudos são sugeridos a fim de 
compreender melhor o quanto os níveis de treinamento e as diferenças metodológicas influenciam nos resultados de desempenho de flexibilidade ao longo do ciclo menstrual.

Deve-se exaltar a não diferença de desempenho entre fases iguais dos dois ciclos menstruais avaliados. Esta deve ser considerada como reafirmação dos resultados encontrados anteriormente, uma vez que o desempenho estabeleceuse de forma semelhante em ambos os ciclos menstruais, indicando que este comportamento é recorrente e diminuindo a probabilidade do resultado ter sido influenciado por qualquer outro fator durante o ciclo menstrual. Além disso, este resultado pode estar relacionado ao baixo volume do protocolo de treinamento aplicado que promoveu pouca melhora de desempenho ao longo do experimento.

Como principais limitações, este estudo apresenta um baixo número de participantes e utiliza apenas um método de avaliação da flexibilidade ao longo dos ciclos investigados. Sob essa perspectiva estudos que avaliem a capacidade de flexibilidade ao longo de mais de um ciclo menstrual e que possuam um protocolo de treinamento ao longo do período experimental, aplicando diferentes métodos de treinamento, são recomendados para contribuir com o entendimento dessa temática.

\section{CONSIDERAÇÕES FINAIS}

A partir dos resultados obtidos conclui-se que as mulheres treinadas em flexibilidade apresentaram maior desempenho ao longo dos ciclos avaliados do que as mulheres sedentárias, porém não foram encontradas diferenças significativas de desempenho em flexibilidade entre as fases de dois ciclos menstruais para mulheres treinadas e sedentárias, bem como no desempenho entre fases iguais destes dois ciclos menstruais.

\section{REFERÊNCIAS}

BISI, Francine Bergamo; DALLE MOLLE, Luise de Oliveira; BARONI, Bruno Manfredini; LEITE, Francine Nardi; BRUSCATTO, Claudia Adriana; JUNIOR, Ernesto Cesar Pinto Leal. Influência do ciclo menstrual na flexibilidade de atletas que utilizam contraceptivo oral. Revista brasileira de ciência e movimento, v. 17, n. 3, p. 18-24, 2009. Disponível em: https://portalrevistas.ucb.br/index.php/RBCM/article/view/1041

CHAVES, Christianne Pereira Giesbrecht; SIMÃO, Roberto; ARAÚJO, Claudio Gil Soares de. Lack of flexibility variation during menstrual cycle in university students, Revista Brasileira de Medicina do Esporte, v. 8, n. 6, p. 212-218, 2002. Disponível em: https://www.scielo.br/scielo.php?pid=S1517-86922002000600002\&script=sci abstract 
GAMA, Zenewton André da Silva; DANTAS, Alexandre Vinícyus Ribeiro; MEDEIROS, Carlos Alexandre de Souza; SOUZA, Túlio Oliveira de. Influence of the stretching frequency using proprioceptive neuromuscular facilitation in the flexibility of the hamstring muscles, Revista Brasileira de Medicina do Esporte, v. 13, n. 1, p. 33-38, 2007. Disponível em: https://www.scielo.br/scielo.php?script=sci arttext\&pid=S1517$\underline{86922007000100008}$

JOHNSON, Richard Arnold; WICHERN, Dean W. Applied multivariate statistical analysis. 6. ed. Upper Saddle River, New Jersey: Prentice hall, v. 5, n. 8, 2002.

LEBRUN, Constance M.; RUMBALL, Jane S. Relationship between athletic performance and menstrual cycle. Current women's health reports, v. 1, n. 3, p. 232-240, 2001. Disponível em: https://pubmed.ncbi.nlm.nih.gov/12112975/

LOUREIRO, Sheila; DIAS, Ingrid; SALES, Dayane; ALESSI, Isabel; SIMÃO, Roberto; FERMINO, Rogério César. Effect of different phases of the menstrual cycle on the performance of muscular strength in 10RM. Revista Brasileira de Medicina do Esporte, v. 17, n. 1, p. 22-25, 2011. Disponível em:

https://www.scielo.br/scielo.php?script=sci arttext\&pid=S1517-86922011000100004

MELEGARIO, Solange Mattos; SIMÃO, Roberto; VALE, Rodrigo G.S.; BATISTA, Luiz Alberto; NOVAES, Jefferson $S$. The influence of the menstrual cycle on the flexibility in practitioners of gymnastics at fitness centers. Revista Brasileira de Medicina do Esporte, v. 12, n. 3, p. 125-128, 2006. Disponível em:

https://www.scielo.br/pdf/rbme/v12n3/en v12n3a03.pdf

TEIXEIRA, André Luiz da Silva; JÚNIOR, Walter Fernandes; MARQUES, Fábio Antônio Damasceno; LACIO, Marcio Luis de; DIAS, Marcelo Ricardo Cabral. Influence of different phases of menstrual cycle on flexibility of young women. Revista Brasileira de Medicina do Esporte, v. 18, n. 6, p. 361-364, 2012. Disponível em:

https://www.scielo.br/scielo.php?script=sci arttext\&pid=S1517-86922012000600002

WELLS, Katharine F.; DILLON, Evelyn K. The sit and reach-a test of back and leg flexibility. Research Quarterly. American Association for Health, Physical Education and Recreation, v. 23, n. 1, p. 115-118, 1952. Disponível em: https://www.tandfonline.com/doi/abs/10.1080/10671188.1952.10761965 